\title{
Daily Serum Collection after Acellular Dermal Matrix-Assisted Breast Reconstruction
}

\author{
Glenda Giorgia Caputo ${ }^{1}$, Zeno Franchini ${ }^{2}$, Monia Maritan ${ }^{1}$, Edoardo Dalla Pozza ${ }^{1}$, \\ Enrico Vigato ${ }^{1}$, Umberto Tedeschi ${ }^{2}$, Maurizio Governa ${ }^{1}$ \\ ${ }^{1}$ Plastic and Reconstructive Surgery, Surgery and Odontology Department, University Hospital of Verona, Verona; ${ }^{2}$ General Surgery, Surgery \\ and Odontology Department, University Hospital of Verona, Verona, Italy
}

Background The acellular dermal matrix (ADM)-assisted breast reconstruction technique is widely known, but discouraging results due to early postoperative complications have been reported. As the literature identifies seroma as the most common issue after breast surgery without identifying its pathogenesis, we aimed to report the trend of postoperative daily serum collection after ADM-assisted breast reconstruction and compare it with data in the literature in order to discover more about this little-known topic.

Methods A retrospective study on 28 consecutive patients who received ADM-assisted breast reconstruction between February 2013 and February 2014 was performed. In order to reduce the number of variables that could affect serum production, only one brand of ADM was used and all tissues were handled gently and precisely. The daily drainage volume was recorded per patient during the first four days of hospitalization. Likewise, postoperative complications were noted during routine follow-up.

Results In total, five (17.9\%) bilateral and 23 (82.1\%) unilateral ADM-assisted breast reconstructions (33 implants) were performed. The mean age, body mass index, and length of hospital stay were 53.6 years, $21.3 \mathrm{~kg} / \mathrm{m}^{2}$, and 4.5 days, respectively. One major complication led to implant loss $(3.0 \%)$, and nine minor complications were successfully treated with ambulatory surgery (27.3\%). Serum collection linearly decreased after 24 hours postoperatively. Conclusions Daily drainage decreased following the theoretical decline of acute inflammation. In concordance with the literature, daily serum production may not be related to the use of ADM.

Keywords Seroma / Acellular dermis / Mammaplasty / Inflammation / Mastectomy
Correspondence:

Glenda Giorgia Caputo

Plastic and Reconstructive Surgery, Surgery and Odontology Department, University Hospital of Verona, Piazzale Stefani 1, Verona, Italy Tel: +39-045-8127603 Fax: +39-045-8122069 E-mail: glendagiorgia.caputo@ ospedaleuniverona.it

Received: 5 Sep 2014 • Revised: 5 Nov 2014 • Accepted: 5 Nov 2014

pISSN: 2234-6163 • elSSN: 2234-6171 • http://dx.doi.org/10.5999/aps.2015.42.3.321 • Arch Plast Surg 2015;42:321-326

\section{INTRODUCTION}

The single-stage, acellular dermal matrix (ADM)-assisted breast reconstruction technique is widely known for its numerous advantages such as a reduced number of surgeries and operative times, improved and reproducible aesthetic results, less postoper- ative pain, and reduced hospital costs [1-3]. However, several studies have reported early postoperative complications, especially seroma formation, for this relatively new technique $[4,5]$. In addition, ADM has been found to increase the incidences of seroma and infection more than fourfold [6]. The development of heavy seroma leads to pain, delayed wound healing, skin flap ne- 
crosis, and infection, thus resulting in prolonged discomfort [7].

However, seroma has been a widely discussed issue even before the use of $\mathrm{ADM}$ in breast reconstruction. Overall, the reported incidence of seroma after any kind of breast surgery varies between $3 \%$ and $81 \%$ [ 8 ].

Nevertheless, the pathogenesis of seroma is poorly understood and remains controversial. Several factors are implicated in seroma formation, including age, body mass index (BMI), neoadjuvant chemotherapy, surgical technique, use of electrocautery, and arm movements soon after surgery [9].

In problematic scenarios, a consensus has not arisen regarding the use of biological matrices to cover the lower pole of the breast in immediate reconstruction. In fact, ADMs can amplify the effects of this postoperative complication, as the fluid layer between the matrix and the mastectomy flap hinders cell migration and the subsequent incorporation of the matrix $[10,11]$, resulting in a failed reconstruction attempt.

To evaluate whether the use of ADM affects or changes the drainage pattern, we analyzed trends in early postoperative daily serum collection after immediate ADM-assisted breast reconstruction and compared these data with those in the literature.

\section{METHODS}

This retrospective study included 28 consecutive patients who received single-stage $\mathrm{ADM}$-assisted breast reconstruction between February 2013 and February 2014. The hospital ethics committee oversaw and approved the study.

Detailed data including patients' demographics, comorbidities, BMI, smoking status, and operative time were collected after a careful review of all patient medical records. Major and minor complications were defined as any early postoperative complication that required a subsequent operation or conservative treatment, respectively. During hospitalization, the volume of serum collected was carefully recorded for each patient daily. In particular, we were interested in comparing serum production collected in this study with that reported in the literature.

Patients were scheduled for routine follow-ups at 7, 15, and 30 days postoperatively as well as 3, 6, 12 months postoperatively.

All breast reconstructions were performed using a single brand non-cross-linked porcine ADM (Native, Medical Biomaterial Products, Neustadt-Glewe, Germany). The Native ADM was 0.6 -mm thick and $18 \times 10 \mathrm{~cm}$, dry, and without any preservatives. The features of this material were designed to increase the performance of the biomaterial and minimize any development of adverse postoperative complications. A preservative free, dry matrix could integrate faster and is less likely to cause an inflammatory reaction than other preservative-based cross-linked bio- materials [12,13].

The breast and plastic surgeons of the Oncoplastic Breast Surgery Team at the University Hospital of Verona collaborate closely to plan and perform mastectomy and breast reconstruction in a single operation. Great importance has been given to reducing the complication rate by carefully tailoring all of the parameters of the surgical technique based on our experiences. For example, we ensure that an adequately sized incision is made to avoid tissue microtrauma due to excessive traction and that electrocautery is not used to minimize tissue damage and discourage the acute inflammatory response $[14,15]$. In addition, povidoneiodine seems to be cytotoxic for fibroblasts and can slow the tissue healing process, thus is not used on breast pockets, breast implants, or matrix irrigation procedures [16,17]. Moreover, surgery time should be kept to a minimum to increase the likelihood of a positive postoperative result [18]. A delicate surgery was preferred, with the aim of reducing the number of factors that can influence postoperative serum production.

Immediately after performing the mastectomy, the subpectoral pocket is created to begin breast reconstruction. The pectoralis major muscle is not detached from its medial insertions; rather, the muscular fibers are detached from only the lateral to medial side and up to the medial fat layer, thus maintaining the attachment of the muscle to the medial fat layer, preserving the muscle, and allowing for minimal retraction. Next, Native ADM is positioned laterally between the lower pole of the breast and the pectoralis major muscle to complete the subpectoral pocket after its hydration. The matrix is first sutured to the inframammary fold, and then to the muscle edge. Then, the matrix is shaped around the breast implant to avoid wrinkling and allow the skin to fit perfectly over the membrane, in order to reduce chronic inflammation and giant-cell foreign-body reactions [19].

Before the cutaneous suture, the edges of incision are trimmed very slightly to expedite the wound healing process and prevent dehiscence and necrosis since the removed tissue is likely to have been ischemically damaged [19].

Last, two drainage tubes, one in the ADM-submuscular pocket and another in the subcutaneous space, are inserted to measure the volume of drainage, and then removed when the drainage reaches $30 \mathrm{~mL}$ or less per day.

All statistical analyses were performed using STATA ver. 12 (StataCorp, College Station, TX, USA).

\section{RESULTS}

Between February 2013 and February 2014, 28 women underwent five (17.9\%) bilateral and 23 (82.1\%) unilateral ADM-assisted breast reconstructions (33 total implants). Among these 
procedures, five (15.2\%) were prophylactic mastectomies, 28 (84.8\%) were curative treatments; 22 (66.7\%) were skin-sparing mastectomies, and 11 (33.3\%) were nipple sparing mastectomies (Table 1).

The mean age was 53.6 years (range, 38 to 69 years), and the mean BMI was $21.3 \mathrm{~kg} / \mathrm{m}^{2}$ (range, 17.3 to $26.4 \mathrm{~kg} / \mathrm{m}^{2}$ ). One patient $(3.0 \%)$ was a current smoker and two $(6.1 \%)$ had received radiotherapy more than 10 years before the surgery. The mean weight of each breast implant was $302.0 \mathrm{~g}$ (range, 135 to $415 \mathrm{~g}$ ). Two drains were used for each breast implant and were removed on average at 7 days postoperatively (range 5 to 10 days postoperatively). The mean length of hospital stay was 4.5 days (Table 1). The total daily serum collected from each drain until 4 days postoperatively is summarized in Table 2 . In addition, Fig. 1 shows a decreasing trend in serum collection with a peak in the first 24 hours (Fig. 1), which follows the theoretical performance of the inflammatory process [20].

Postoperative data was analyzed for each patient. One (3.0\%) major postoperative complication that lead to implant loss was found and was likely due to an untreated dehiscence that became infected. In addition, two (6.1\%) hematomas and seven

\section{Table 1. Demographic and clinical data}

\begin{tabular}{|lc|}
\hline Characteristic & Data \\
\hline Patients (no.) & 28 \\
Age (yr) & $53.61 \pm 9.86(38-69)$ \\
Body mass index $\left(\mathrm{kg} / \mathrm{m}^{2}\right)$ & $21.22 \pm 2.49(17.36-26.37)$ \\
Smokers & $1(3.57)$ \\
Previous radiotherapy & $2(7.14)$ \\
Total implants (no.) & 33 \\
Unilateral implants & $23(82.14)$ \\
Bilateral implants & $5(17.90)$ \\
Prophylactic mastectomy & $5(15.15)$ \\
Curative mastectomy & $28(84.85)$ \\
Skin sparing mastectomy & $22(66.67)$ \\
Nipple sparing mastectomy & $11(33.33)$ \\
Average of breast implant (mL) & $302.04 \pm 87.30(135-450)$ \\
Mean hospital stay (day) & $4.50 \pm 1.47(4-10)$ \\
Mean time of drains removal (day) & $7.00 \pm 1.66(5-10)$ \\
\hline Values are presented as number (\%) or mean \pm standard deviation (range). \\
\hline
\end{tabular}

Table 2. Average drainage during the first 4 days postoperatively

\begin{tabular}{|lcc|}
\hline Day & Drain $\mathbf{1} \pm \mathrm{SD}(\mathrm{mL})$ & Drain $2 \pm \mathrm{SD}(\mathrm{mL})$ \\
\hline 1 & $153.6 \pm 39.44$ & $54.5 \pm 37.78$ \\
2 & $92.3 \pm 34.81$ & $29.4 \pm 16.24$ \\
3 & $47.7 \pm 21.26$ & $18.5 \pm 13.65$ \\
4 & $33.2 \pm 25.33$ & $12.3 \pm 11.24$ \\
\hline $\begin{array}{l}\text { Drain 1, in the acellular dermal matrix-submuscular pocket; SD, standard devia- } \\
\text { tion; Drain 2, in the subcutaneous position. }\end{array}$ \\
\hline
\end{tabular}

(21.2\%) cases of dehiscence were successfully treated with wound-edge debridement and sutures (Table 3). Our singlestage $\mathrm{ADM}$-assisted breast reconstruction resulted in symmetrical breasts with natural ptosis and a natural aesthetic (Fig. 2).

At 2 months postoperatively, a wound dehiscence was treated at the outpatient clinic, and the ADM-implant site was biopsied for histologic evaluation. The biopsy, stained with hematoxylin and eosin, showed fibroblast ingrowth and complete vascularization, evidence that the matrix had become incorporated (Fig. 3).

\section{DISCUSSION}

Although our experience is limited, we found a low rate of postoperative complications after ADM-assisted breast reconstruction. Sbitany and Serletti [5] and Chun et al. [6] reported discouraging results; nevertheless, the $\mathrm{ADM}$ has been found to have many advantages over the traditional expander technique, such as reduced health care costs, physical and psychological suffering of the patients, and overall number of procedures $[2,3]$. In addition, cosmetic outcomes are improved by inserting the $\mathrm{ADM}$ sling into the lower pole maintaining a natural shape with the correct projection in the lower part of the operated breast, all of which was obtained in a single operative session [3]; overall, good aesthetic results were also observed in our

\section{Fig. 1. Postoperative daily serum collection}

The average volume of serum collected daily from the acellular dermal matrix-submuscular pocket (drain 1) and the subcutaneous position (drain 2) during the first 4 days postoperatively.

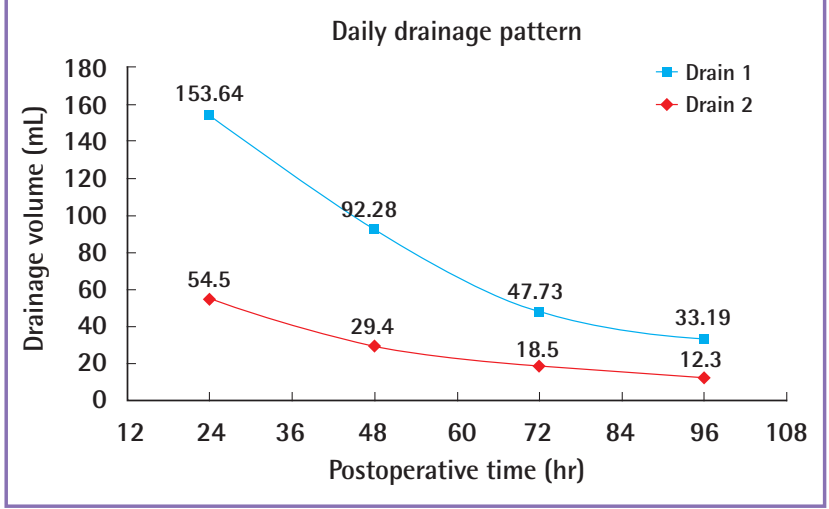

\section{Table 3. Complication rates}

\begin{tabular}{|lll|}
\hline $\begin{array}{l}\text { Type of } \\
\text { complications }\end{array}$ & \multicolumn{1}{c|}{ Description } & No. (\%) \\
\hline Major ${ }^{\text {a) }}$ & Infections (Staphylococcus aureus) & $1(3.0)$ \\
Minor) $^{b}$ & Hematomas & $2(6.1)$ \\
& Dehiscences & $7(21.2)$ \\
\hline $\begin{array}{l}\text { a)Major complications required the return to the theater for the implant removal; } \\
\text { b)Minor complications were treated conservatively. }\end{array}$ \\
\hline
\end{tabular}




\section{Fig. 2. Aesthetic outcomes}

Results of a skin-sparing mastectomy with an acellular dermal matrix-assisted reconstruction on the left breast. Preoperative pictures in the lateral and frontal views (AC) with the foreground of the original ptosis of the left breast (D) and postoperative lateral and frontal pictures at 30 days (E-G) are shown. Postoperatively, preservation of the natural ptosis was achieved $(H)$.
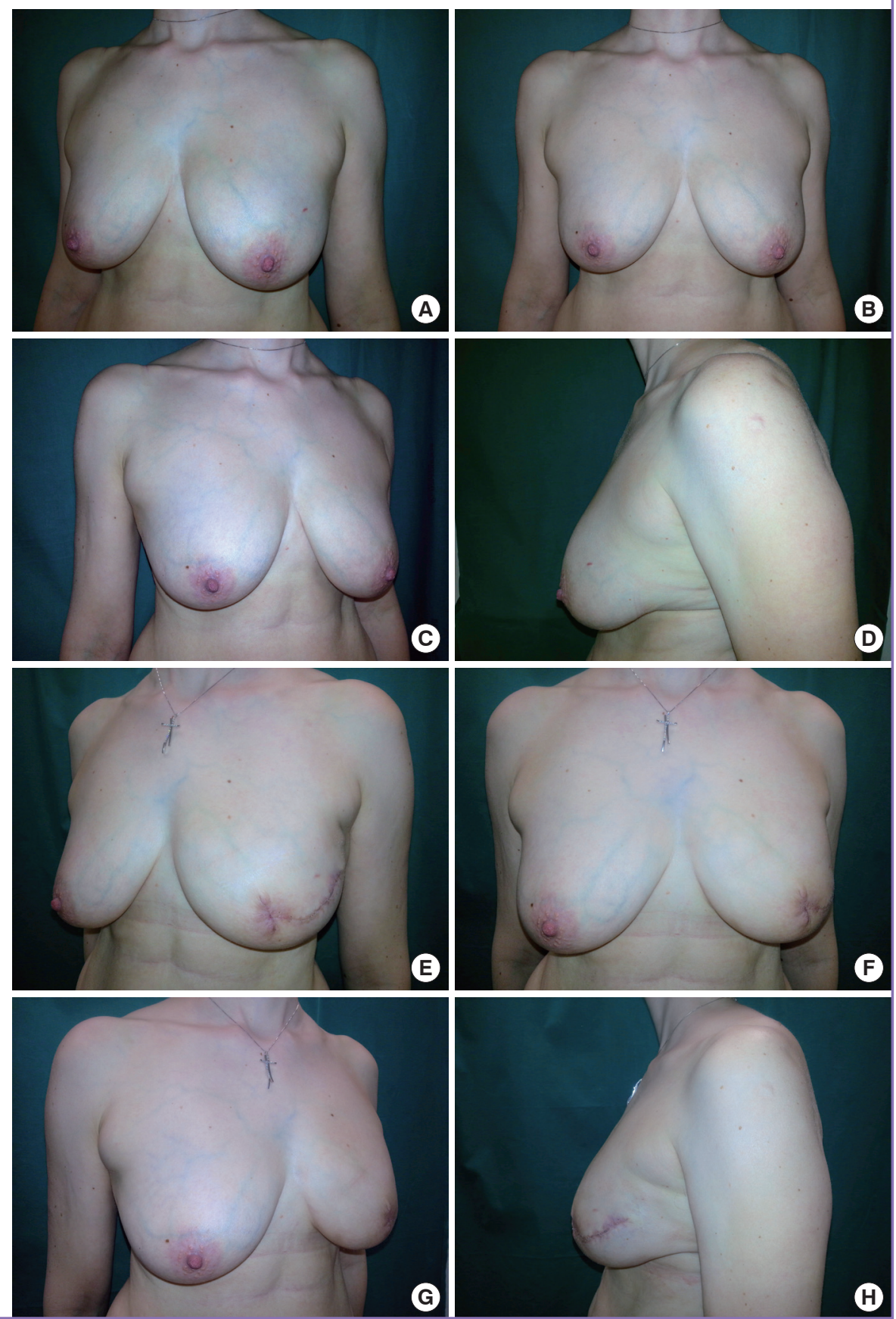

study (Fig. 2).

A high incidence of postoperative complications such as large seromas have been attributed to the use of ADM and often become the origin of further major complications [5]. Although not life threatening, seromas may increase the risk of wound dehiscence, infections, skin-flap necrosis, a subsequent prolonged recovery period, and multiple outpatient examinations [7]. However, in cases of mastectomy without reconstruction, the incidence of seroma was reported to be as high as $85 \%$ [8].

Although many studies have suggested guidelines for the treat- ment and prevention of seromas [11], the etiology of this wellknown problem remains unknown. In fact, the pathological origin of seromas has not been fully clarified, but has been found to be associated with several factors such as age, BMI, breast size [9], the type of surgical procedure [10], and the use of electrocautery [14]. However, several studies have demonstrated the inflammatory origin of the exudate; it seems likely to be the body's natural response to tissue damage [7-9,21,22]. Therefore, our Oncoplastic Breast Surgery Team decided to perform a conservative and delicate surgical technique aimed at reducing 


\section{Fig. 3. Capsular histological samples 2 months postoperatively}

The microscopic view demonstrates complete incorporation of the matrix with large cell and microvascular ingrowth $\left(H \& E_{1} \times 100\right)$.

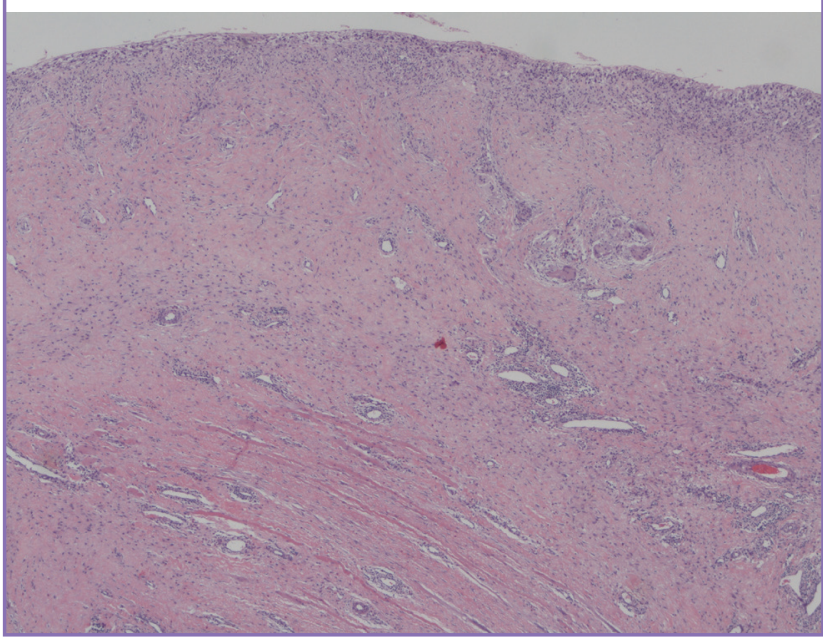

tissue trauma in hopes of attenuating the inflammatory response and reduce the variables related to serum production. For this reason, any element thought to potentially increase the inflammatory response was avoided for a more reliable assessment of the influence of $\mathrm{ADM}$ in the development of this postoperative complication. Electrocautery was used minimally and replaced with the use of a cold scalpel and surgical scissors to avoid thermal trauma [14]. In addition, povidone-iodine was not used to rinse the breast implant, $\mathrm{ADM}$, or breast pocket because this antimicrobial is toxic for fibroblasts and may have detrimental effects on wound healing [16]. Moreover, a non-cross-linked porcine collagen matrix (Native, Medical Biomaterial Products, Neustadt-Glewe, Germany) was used. This biocompatible material, dry and without any preservatives, does not affect the natural process of tissue repair and minimizes or greatly reduces the likelihood of an adverse body reaction $[12,13,23]$.

Seroma development in ADM-assisted breast reconstruction complicates the matrix incorporation by the presence of a fluid layer between the mastectomy flap and the acellular matrix, which can slow cell and microvascular ingrowth and delay incorporation of the graft [10]. Therefore, $\mathrm{ADM}$ is often related to a high percentage of postoperative complications. For this reason, Brzezienski et al. [11] proposed an algorithm for the management of postoperative seromas after immediate breast reconstruction using an $\mathrm{ADM}$ in order to minimize implant loss and optimize outcomes. However, the solution seemed to reduce the number of $\mathrm{ADM}$-assisted breast reconstructions, without solving the underlying problem.

Our study aimed to examine the quantity of drainage after $\mathrm{ADM}$-assisted breast reconstruction to compare it with the quantity of drainage after mastectomy without reconstruction
[24], as reported in the literature, in order to assess whether the use of $\mathrm{ADM}$ increases or changes the amount of postoperative serum production and its pattern over time.

Fig. 1 indicates that serum production peaked at 24 hours postoperatively and then rapidly declined thereafter; this trend is similar to that of the theoretical course of the acute inflammatory response [20]. Therefore, our results support the pro-inflammatory theory of seromas; they seem to be associated with the acute phase of wound healing after a surgical procedure [21]. Our data revealed a daily serum collection trend (Fig. 1) very close to that of the daily drainage patterns reported after mastectomy only [24]. Although the serum production in our patients was less than that in the previous study [24], the two trends were very similar. Therefore, the use of $\mathrm{ADM}$ seems to have no effect on the drainage pattern previously believed to be due to surgical trauma, but instead seems to follow the development of inflammatory response.

No heavy seromas were found in any of our 28 patients who received ADM-assisted breast reconstruction; all women followed a normal postoperative course of seroma production that steadily decreased in the four days postoperatively. In addition, all patients returned to the hospital for drain removal within 10 days postoperatively, and no patient required serum aspiration. We observed only one major complication (3.0\%) due to an untreated dehiscence that was exacerbated by infection.

A sample of the $\mathrm{ADM}$ was obtained at two months postoperatively, and histological analysis revealed a complete integration of the matrix with the patient's dermis as also found in other similar articles $[3,10]$.

These findings suggest that the ADM may not be involved in seroma formation.

For a successful seroma-free ADM-assisted breast reconstruction, based on our experience, we could suggest a surgical technique aimed at reducing the postoperative inflammatory response and the use of a "low irritative" and highly biocompatible medical device like a dry, preservative-free, non-cross-linked $\operatorname{ADM}[12,13]$.

In conclusion, seroma remains an unresolved issue that can result in serious postoperative complications. Considering the results of our study and that of previous studies, serum production seems to be a natural consequence and a "necessary evil" of the inflammatory reaction after tissue damage [25], and the use of an $\mathrm{ADM}$ for immediate breast reconstruction may not increase serum collection.

Nevertheless, these results should be validated by future, prospective, multi-institutional investigations. 


\section{REFERENCES}

1. Salzberg CA, Ashikari AY, Koch RM, et al. An 8-year experience of direct-to-implant immediate breast reconstruction using human acellular dermal matrix (AlloDerm). Plast Reconstr Surg 2011;127:514-24.

2. Johnson RK, Wright CK, Gandhi A, et al. Cost minimisation analysis of using acellular dermal matrix (Strattice) for breast reconstruction compared with standard techniques. Eur J Surg Oncol 2013;39:242-7.

3. Zienowicz RJ, Karacaoglu E. Implant-based breast reconstruction with allograft. Plast Reconstr Surg 2007;120:37381.

4. Hoppe IC, Yueh JH, Wei CH, et al. Complications following expander/implant breast reconstruction utilizing acellular dermal matrix: a systematic review and meta-analysis. Eplasty 2011;11:e40.

5. Sbitany H, Serletti JM. Acellular dermis-assisted prosthetic breast reconstruction: a systematic and critical review of efficacy and associated morbidity. Plast Reconstr Surg 2011; 128:1162-9.

6. Chun YS, Verma K, Rosen H, et al. Implant-based breast reconstruction using acellular dermal matrix and the risk of postoperative complications. Plast Reconstr Surg 2010;125: 429-36.

7. Kuroi K, Shimozuma K, Taguchi T, et al. Evidence-based risk factors for seroma formation in breast surgery. Jpn J Clin Oncol 2006;36:197-206.

8. Srivastava V, Basu S, Shukla VK. Seroma formation after breast cancer surgery: what we have learned in the last two decades. J Breast Cancer 2012;15:373-80.

9. Zielinski J, Jaworski R, Irga N, et al. Analysis of selected factors influencing seroma formation in breast cancer patients undergoing mastectomy. Arch Med Sci 2013;9:86-92.

10. Michelotti BF, Brooke S, Mesa J, et al. Analysis of clinically significant seroma formation in breast reconstruction using acellular dermal grafts. Ann Plast Surg 2013;71:274-7.

11. Brzezienski MA, Jarrell JA 4th, Mooty RC. Classification and management of seromas in immediate breast reconstruction using the tissue expander and acellular dermal matrix technique. Ann Plast Surg 2013;70:488-92.

12. Carlson TL, Lee KW, Pierce LM. Effect of cross-linked and non-cross-linked acellular dermal matrices on the expression of mediators involved in wound healing and matrix re- modeling. Plast Reconstr Surg 2013;131:697-705.

13. Mestak O, Spurkova Z, Benkova K, et al. Comparison of cross-linked and non-cross-linked acellular porcine dermal scaffolds for long-term full-thickness hernia repair in a small animal model. Eplasty 2014;14:e22.

14. Porter KA, O'Connor S, Rimm E, et al. Electrocautery as a factor in seroma formation following mastectomy. Am J Surg 1998;176:8-11.

15. Ozdogan M, Yilmaz KB, Ozaslan C, et al. Scalpel versus electrocautery dissections: the effect on wound complications and pro-inflammatory cytokine levels in wound fluid. Turk J Med Sci 2008;38:111-6.

16. Van den Broek PJ, Buys LF, Van Furth R. Interaction of povidone-iodine compounds, phagocytic cells, and microorganisms. Antimicrob Agents Chemother 1982;22:593-7.

17. Adams WP Jr, Conner WC, Barton FE Jr, et al. Optimizing breast pocket irrigation: an in vitro study and clinical implications. Plast Reconstr Surg 2000;105:334-8.

18. Salzberg CA. Direct-to-implant breast reconstruction with Acellular Dermal Matrix. In: Spear SL, Robb GL, Hammond DC, et al., editors. Surgery of the breast: principles and art. 3rd ed. Philadelphia: Wolters Kluwer/Lippincott Williams \& Wilkins; 2010. p.412-9.

19. Ganske I, Verma K, Rosen H, et al. Minimizing complications with the use of acellular dermal matrix for immediate implant-based breast reconstruction. Ann Plast Surg 2013; 71:464-70.

20. Lawrence T, Willoughby DA, Gilroy DW. Anti-inflammatory lipid mediators and insights into the resolution of inflammation. Nat Rev Immunol 2002;2:787-95.

21. Szecsi PB, Larsen J, Horby J, et al. Seroma production after breast cancer surgery has a pro-inflammatory component. Open Breast Cancer J 2012;4:11-7.

22. Sampathraju S, Rodrigues G. Seroma formation after mastectomy: pathogenesis and prevention. Indian J Surg Oncol 2010;1:328-33.

23. Badylak SF. Decellularized allogeneic and xenogeneic tissue as a bioscaffold for regenerative medicine: factors that influence the host response. Ann Biomed Eng 2014;42:1517-27.

24. Lin YP, Yin WJ, Yan TT, et al. Risk factors for postoperative seromas in Chinese breast cancer patients. Chin Med J (Engl) 2011;124:1300-4.

25. Gonzalez EA, Saltzstein EC, Riedner CS, et al. Seroma formation following breast cancer surgery. Breast J 2003;9:385-8. 